\title{
Es tut mir leid, Dave, das kann ich nicht tun ...
}

... sagt der Bordcomputer in Stanley Kubricks Science-Fiction-Klassiker „2001: Odyssee im Weltraum“ - sicher eine der bekanntesten Szenen der Filmgeschichte, in der Mensch und Maschine kommunizieren. Gleichzeitig steht sie für die Sorge, Maschinen könnten so intelligent werden, dass sie für den Menschen zur Gefahr werden. Maschinen, die kommunizieren, werden oft auch als etwas unheimlich wahrgenommen. Im echten Leben ist die Kommunikation mit Maschinen längst Alltag, teilweise findet sie so statt, dass wir das nicht einmal bewusst wahrnehmen, aber die (nahe) Zukunft verspricht noch viel mehr.

Große Fortschritte macht beispielsweise die - in Kubricks Film vorweggenommene - Kommunikation über das gesprochene Wort. In vielen Haushalten werden schon heute Alexa und Co. genutzt, die gesprochene Fragen beantworten oder etwa auf Wunsch das Licht anschalten. Das funktioniert nicht immer perfekt, aber doch bereits recht gut. Herausforderungen gibt es eindeutig beim Thema Datenschutz, da die gesprochenen Worte aufgenommen und in der Cloud verarbeitet werden. Damit haben die Anwender quasi eine Wanze im Haus installiert. Trotzdem kann man davon ausgehen, dass schon bald immer mehr kommunikationsfähige Maschinen in unseren Haushalten Einzug halten werden. Ein wichtiger Nutzen besteht darin, dass mithilfe dieser Geräte ältere Menschen länger unabhängig zu Hause wohnen werden können.

Auch im beruflichen Umfeld ist Mensch-Maschinen-Kommunikation nicht mehr wegzudenken und die Möglichkeiten sind bei Weitem noch nicht ausgeschöpft. Dabei sind vor allem unterschiedliche Arten von Assistenzsystemen von Bedeutung. Der Mensch wird dabei durch die Technik oft nicht ersetzt, sondern unterstützt oder es wird eine Funktion, die zurzeit ein Mensch ausführt, von einem Roboter übernommen, dieser arbeitet aber mit Menschen im Team, mit denen er dann effizient kommunizieren muss. Entscheidend ist dabei, dass die Roboter keine Gefahr für die Menschen in ihrer Umgebung darstellen dürfen.

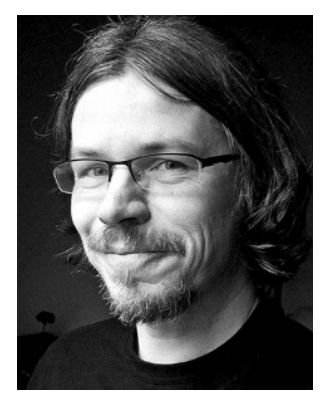

Peter Pagel $(\bowtie)$

peter.pagel@springer.com

Wiesbaden, Deutschland

Ob im Privaten oder im Beruflichen, Sicherheit und Datenschutz müssen gewährleistet sein, wenn die beschriebenen Szenarien verstärkt Wirklichkeit werden sollen. Sonst droht mangelnde Akzeptanz und eine neue Generation von Maschinenstürmern, die Kollege Computer und Roboter fundamental ablehnen - was hieße, das Kind mit dem Bade auszuschütten.

Herzliche Grüße

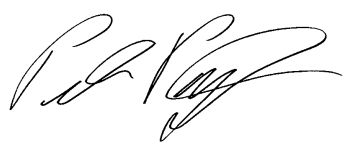

Peter Pagel

Wirtschaftsinformatik \& Management 2019 • 11 (3): 121 https://doi.org/10.1365/s35764-019-00189-3

(c) Springer Fachmedien Wiesbaden GmbH, ein Teil von Springer Nature 2019 